\title{
Determining interstellar dust properties with scattered X-ray halo
}

\author{
Zhixing Ling ${ }^{1}$ and Shuang Nan Zhang ${ }^{2,1}$ \\ ${ }^{1}$ National Astronomical Observatories, Chinese Academy of Sciences, Beijing, China 100012 \\ ${ }^{2}$ Key Laboratory of Particle Astrophysics, Institute of High Energy Physics, Chinese Academy of Sciences, Beijing, China 100049
}

(Received September 7, 2010; Revised February 3, 2011; Accepted February 7, 2011; Online published February 2, 2012)

\begin{abstract}
X-ray photons, emitted by X-ray sources, are absorbed and scattered by dust grains when they travel through interstellar medium. The scattered photons within small angles result in a diffuse X-ray "halo". Therefore, the scattered X-ray photons carry information about various properties of the dust. We study the light curves of the dust-scattered X-ray halo of Cyg X-1. Significant time lags are found between the light curves of the point source and its halo. This time lag increases with the angular distance. This can be caused by a dust concentration at $1.75 \mathrm{kpc}$ from the Earth along the line of sight (LOS), with a width of $\Delta L=33 \mathrm{pc}$. The time lag of Cyg X-3 also reveals a dust concentration between the point source and the Earth, and the distance of Cyg X-3 is estimated to be about $7.2 \mathrm{kpc}$. The origin of these dust concentrations is still not clearly known. Along with recent results on the dust halos of Gamma Ray Bursts (GRBs), we conclude that the dust distribution is quite nonuniform. We also study dust properties with an expanding X-ray ring from the observation of a distant GRB. The distribution of the halo photons can be used to determine the dust-radius model. Our preliminary results show that dust grain geometries may vary significantly across the Galaxy. We also propose to use the scattered X-ray halo to study the composition of the dust grains. The applicability of this method needs to be tested with advanced instruments in the future.
\end{abstract}

Key words: Scattering, halo, dust grain, Cyg X-1, Cyg X-3, GRB.

\section{Introduction}

The existence of a dust-scattered X-ray halo was first proposed by Overbeck (1965) theoretically. In that work, he pointed out that such a halo could provide valuable information about the interstellar dust grains and the typical dust scattering angle was predicted to in the range of several arcminutes to a few degrees. During the following twenty years, further calculations and applications were carried out. Because the small scattering angle results in a small observational angle, such a halo was not observed until the launch of the Einstein observatory, which has an angular resolution of several arcminutes. Rolf (1983) first observed the halo phenomenon by analyzing the data of GX 339-4 with the IPC instrument onboard the Einstein Observatory. Since then, more and more dust-scattered halos have been found in X-ray observations. Predehl and Schmitt (1995) analyzed the observational data of the ROSAT Observatory and found most of those sources have X-ray halos; the halo fractions varied from a few percent to tens of percent. They also found that the effective scattering optical depth is proportional to the visual extinction. The launch of the Chandra X-ray Observatory and XMM-Newton X-ray Observatory have provided a new opportunity to study the properties of such halos more precisely. With the ACIS-I instrument onboard the Chandra Observatory, Yao et al. (2003) resolved the halo surface brightness to even less than 1 arc-

Copyright (C) The Society of Geomagnetism and Earth, Planetary and Space Sciences (SGEPSS); The Seismological Society of Japan; The Volcanological Society of Japan; The Geodetic Society of Japan; The Japanese Society for Planetary Sciences; TERRAPUB.

doi:10.5047/eps.2011.02.004 sec. Xiang et al. (2005) analyzed the Chandra observations of $17 \mathrm{X}$-ray sources and found that nearly all of the halos require that the dust is concentrated near the source.

During recent years, many Gamma Ray Bursts (GRBs) have shown expanding rings around the sources of the images. These rings were confirmed to be photons scattered by the concentrated dust grains. Some observations even showed that more than one ring existed near the sources, which indicated that there was more than one dust concentration along the line of sight (LOS) of a GRB (Vaughan et al., 2004; Tiengo et al., 2010). There are mainly two different ways to study the X-ray halos. The first is the delay property of the scattered photons. Because scattered photons travel through longer paths than unscattered photons, they must lag those unscattered photons. The length of the lag is related to both the location where the scattering takes place and the distance of the source. The exact scattering cross-section and the dust-grain properties are not important in this method. Trümper and Schönfelder (1973) and Hu et al. (2004) have also proposed to use the smeared property of the light curves to study the scattered halo. Because of the bad statistics in the data of the actual observations, these methods have not been tested so far. The other method is the halo-brightness distribution. Nearly all X-ray scattered halos are found by comparing the observed radial surface brightness with the point spread function (PSF) of the telescope used. The total fraction of the scattered X-ray photons can be derived by this method precisely. In summary, because the scattering process is related to the properties of the dust grains, i.e. the space distribution, the chemical composition, and the radii of the dust grains, we can study 
those properties with the X-ray scattered halos.

In Section 2.1, we present the nonuniform dust distribution found by studying X-ray scattered halos. The study of GRB031203 is shown in Section 2.2. A summary and discussions are presented in Section 3.

\section{Method to Derive the Property of Dust Grains 2.1 The nonuniform spatial distribution of interstellar dust}

Figure 1 is an illustration of the scattering process. The $\mathrm{X}$-ray source and observer are located at $x=1$ and $x=0$, respectively. The scattering event happens at $x$ (between 1 and 0 ). The scattering and observational angles in Fig. 1 are $\theta$ and $\phi$, respectively. From those parameters, the lag time of scattered photons at $x$ can be expressed as

$t_{\text {Delay }}(\phi, x)=\left(\frac{x}{\cos \phi}+\sqrt{(1-x)^{2}+(x \tan \phi)^{2}}-1\right) \times \frac{D}{c}$.

Figure 2 shows the typical lag time vs the scattering location $x$ at observational angles of $10^{\prime \prime}, 30^{\prime \prime}, 60^{\prime \prime}$, and $120^{\prime \prime}$, respectively. From Fig. 2, the typical lag time is in the kilosecond range, which can be observed by the Chandra and Xmm-Newton observatories easily.

Predehl et al. (2000) first analyzed the Chandra observations of the light curves of Cyg X-3 and found an obvious lag in the energy band of 0.8 to $2 \mathrm{keV}$. With those light curves and assuming a uniform dust distribution along the LOS, they gave a distance estimation of Cyg X-3 as $9 \mathrm{kpc}$, which is consistent with distance estimations using other methods.

Smith et al. (2002) analyzed the Chandra observation of GX $13+1$. By subtracting the PSF, they gave a precise halo surface brightness between $50^{\prime \prime}$ to $600^{\prime \prime}$. The fractional halo intensity distribution agrees well with theoretical predictions. They fit the halo surface brightness with two different dust distributions: The first assumes a uniform distribution along the LOS, and the second has four dust clouds located at $x=0.2, x=0.4, x=0.6$ and $x=0.8$, respectively. Both dust distributions can fit the data. Xiang et al. (2005) reconstructed the halo surface brightness at $1^{\prime \prime}$. The reconstructed results indicated that most of the dust was concentrated near the source. Ling et al. (2009a) analyzed the Chandra observations of Cyg X-1 and found an obvious time lag in the data of OBSID 3814. Figure 3 shows the light curves of the halo as well as that of the source together. The light curve of the source is derived from the streak line of the observation, which is not affected by the pile-up effect. The bin width of the light curve of the halo is $5^{\prime \prime}$. Besides the time-lag property between the light curve of the X-ray source and that of the halo, we also find that the profile of the light curves of the halo is quite similar to the light curve of the X-ray source. By assuming the distance of Cyg X-1 to be $2.0 \mathrm{kpc}$, the increasing time lag at an increased observational angle indicates a dust concentration at a distance of $1.75 \mathrm{kpc}$ from us. A similar profile property implies that the dust concentration must be quite small in the LOS; otherwise the light curves of the halo would have been smoothed significantly. A monte carlo simulation shows that a depth of $33 \mathrm{pc}$ of the dust concentration along

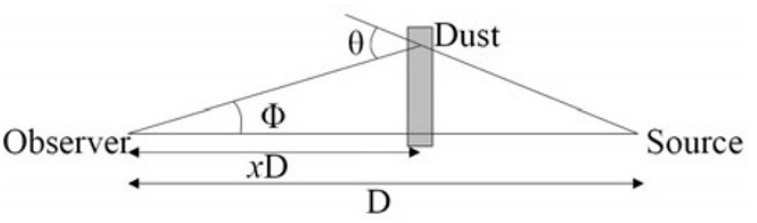

Fig. 1. X-ray dust scattering geometry.

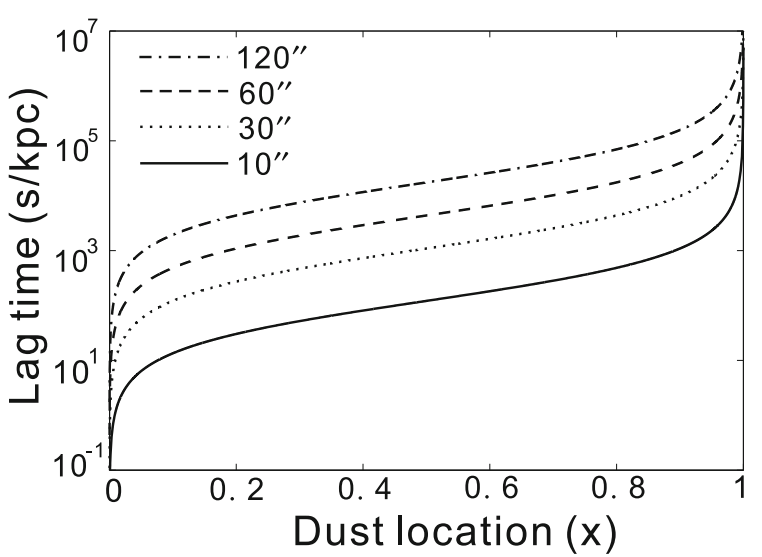

Fig. 2. Lag time vs the scattering location $x$ at different observational angles. All curves are calculated from Eq. (1) with the distance $D$ to be $1 \mathrm{kpc}$.

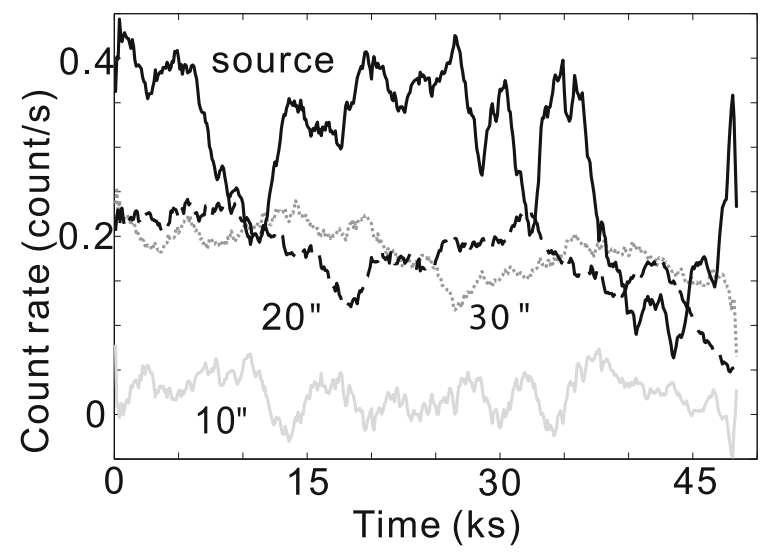

Fig. 3. Light curves of Cyg X-1 and its halo. The solid line is the light curve of the source, derived from the streak line of the observation. The gray solid line shows the light curve of the halo at 10 arcsec away from the source. The other two dashed lines show the light curves at 20 arcsec and 30 arcsec, respectively. The bin width is 5 arcsec and all of the light curves are smoothed with a window of 2 kiloseconds. PSF contaminations have been removed in the light curves of the halo.

the LOS to Cyg X-1 can reproduce the observed light-curve patterns (Ling et al., 2009a). After determining the nonuniform distribution of Cyg X-1, we reanalyzed the data of Cyg X-3 in 2009 (Ling et al., 2009b) and estimated a new distance of about $7.2 \mathrm{kpc}$, with a model consisting of a uniform dust distribution and a dust concentration at the distance of $1.7 \mathrm{kpc}$.

During recent years, many GRB observations show evolutional circles around the sources. For example, Vaughan 
et al. (2004) found two expanding bright rings around GRB031203. Those two circles indicate two dust concentrations along the LOS. They claimed that the distances of these two dust walls are $882 \mathrm{pc}$ and $1388 \mathrm{pc}$, respectively. The width of the dust walls along the LOS was also claimed to be in the range of several parsecs, similar to our result for Cyg X-1. For GRBs, the parameter $x$ can be set to 0 because the GRBs are in the cosmological distance and we can only study the dust in the vicinity of the Sun because the limitation of the observed lag time. The lag time of the halo photons can be solved from Eq. (2),

$$
t_{\mathrm{GRB} \text { Delay }}(\phi, d)=\left(\frac{d}{\cos \phi}-d\right) \times \frac{1}{c},
$$

here, the dust is located at distance $d$, different from Eq. (1).

According to the results above, we conclude that the dust distribution in our galaxy can be probed with the help of Xray scattered halos. The dust is nonuniform in our galaxy and mainly concentrated with sizes of tens of pc. The physics of this concentration is still not clear.

\subsection{The geometric model of dust grain}

In the previous section, we have mentioned that many expanding circles were found around GRB sources and delayed by several kiloseconds after the detection of a GRB. Those bright circles come from the dust-scattered photons, which carry information concerning the dust grains. The incident direction of the scattered photons can be treated as being parallel in Fig. 1.

The location of the scattering dust can be calculated using Eq. (2). Because typically an X-ray observatory can only observe a GRB several kiloseconds after the detection of a GRB with a typical observation time of kiloseconds, we can only study the dust in the nearby several kilo parsecs from the Earth in the direction of the GRB. The distanceestimation methods have been developed by Vaughan et al. (2004), and Tiengo and Mereghetti (2006). In the study of the dust-scattered halo of a GRB, the X-ray light curve of the source can be treated as an ideal delta function because a GRB duration is typically in the range of several seconds, a negligible value compared to the time span of observations and the travel time in the interstellar medium. The observed light curves of the halos at different observational angles are thus just the realizations of the response function of a delta signal. Because the same incident direction is considered and the assumption of the same dust grain at the location of dust concentration, the observed halo surface brightness at different angles is proportional to the scattering crosssection of the dust grain. As discussed by Overbeck (1965), the scattering cross-section is not very sensitive to the shape of the dust grain. This provides a possible way for testing the dust-grain model with the halo surface brightness of the GRB observations.

In this study, we use the dataset of GRB031203, which has the most scattered photons among those GRB observations with obvious halos. GRB031203 was observed at 04:09:29 UT on December 4, 2003, by the XMM-Newton X-ray Observatory 20 kiloseconds after the GRB detection. We extract the photons near the GRB source in the energy band below $2.5 \mathrm{keV}$ with SAS version 7.3. Because the scattering is more efficient in the low-energy band, we only

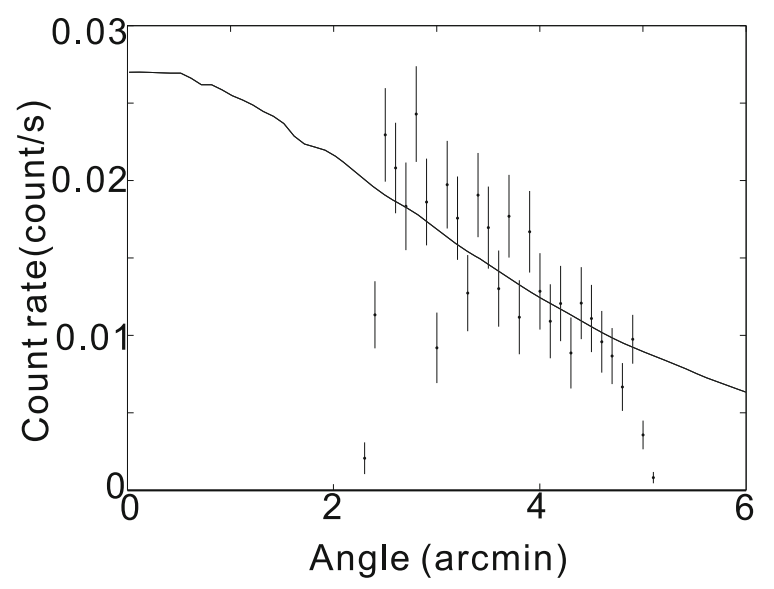

Fig. 4. The model line and the observed angular distribution of GRB031203.

use those low-energy photons if not otherwise indicated. After subtracting the background photons, we can extract the halo surface brightness at different observational angles around the source directly with the scattered photons at a distance of $882 \pm 20$ pc. As shown in Fig. 4, every observed photon could give a parameter called "scattering dust distance". We choose photons with this parameter having a value in the range $822 \mathrm{pc}$ to $942 \mathrm{pc}$. These values are chosen with three confidence intervals. The background photon distribution is taken to be those photons far away from the GRB location. As shown in Fig. 4, the solid line shows the processed and cleaned halo surface distribution of the actual observations. As discussed above, the observed halo surface brightness of the GRB observation is predicted to be proportional to the cross-section of the dust grain theoretically; however the real observational efficiency is degraded at larger angles away from the focus because of the grazing process. Finally, we multiply the effective area of the MOS detector onboard the XMM-Newton observatory and the cross-section of the dust grain model proposed by Weingartner and Draine (2001) (WD2001 hereafter) to represent the "theoretical" prediction of the halo surface brightness. We indicate the result of this model in Fig. 4 by the solid line. The model fits the observed data quite well. This result shows that the dust grain model of WD2001 is adequate to explain the halo surface brightness of GRB031203 for scattering dust at the distance of $882 \mathrm{pc}$.

In comparison, during the study of Cyg X-1 and Cyg X3 (Ling et al., 2009a, b), we found that the WD2001 dust grain model does not fit the observed data of halo surface brightness. Combining the GRB result, we propose that the dust grains in different directions and at different locations in our galaxy can be quite different. The scattering halo provides a new way to study the geometric distribution of dust grains in our galaxy. Further research is in progress.

\subsection{The chemical composition of dust grains}

The chemical composition of dust grains and the dustgas ratio are crucial parameters in the study of interstellar medium. The presence of dust is generally inferred by depletion in the UV and optical bands. Because both gas and dust are semitransparent in X-rays, the measured spectral 
absorption in the X-ray band is sensitive to all atoms in both the gas and solid phase. Lee and Ravel (2005) have proposed the viability of using X-ray absorption fine structure to detect the state and composition of interstellar dust grains with calorimeters onboard the Suzaku observatory. Due to the inflight failure of the calorimeter, the study of the absorption fine structure cannot be made for energies higher than $6 \mathrm{keV}$. Therefore Lee et al. (2009) proposed a new technique to study the absorption property of the Xray spectra in the soft X-ray band. Because of the different scattering cross-section of the dust and gas, the absorption fine features can be decomposed into two components (see section 3.2 of Lee et al. (2009) and the references therein). Lee et al. (2009) gave a preliminary result for Cyg X-1, which shows a good spectral decomposition. In addition to the spectrum of the X-ray source, the simultaneously observed scattering halo can be used to decompose the spectrum with the same method. This halo result yielded information about the interstellar medium. However, some problems must be solved before the application of this analysis to the X-ray scattered halo. The first is the precise measurement of the cross-sections and the normalization of the absolute value, as discussed in Lee et al. (2009). The second is the requirement of more powerful instruments, with high-energy resolution and a larger effective area, such as the International X-ray Observatory.

\section{Summary}

In this work, we have summarized the observed data of X-ray binaries and GRB031203 on the phenomena of Xray scattering by dust. We propose that the dust scattered halo can be used to determine the properties of interstellar dust grains. By studying the light curves of the halo of Cyg X-1, we found a dust concentration at a distance of $1.75 \mathrm{kpc}$ from the Earth. The time lag of Cyg X-3 also indicates a dust concentration along the LOS. The study of GRB observations also show a dust concentration in the vicinity of the Sun. All of these results imply that the dust distribution in our galaxy is nonuniform.

The geometrical dust-grain model can also be determined by the surface brightness distribution of the halos. A simple study of Cyg X-1 and Cyg X-3 shows that the WD2001 model does not fit the data of halo surface brightness. We also use GRB031203 to test the WD2001 dust-grain models. A preliminary result shows that the WD2001 model does fit the GRB data well. We thus conclude that the dustgrain geometric properties may differ significantly at different locations in our galaxy; however, in previous studies a simple and universal dust-grain model has always been assumed for the entire Galaxy. Following the method of
Lee and Ravel (2005) and Lee et al. (2009), the chemical composition of dust grain can also be estimated by studying X-ray scattered halos.

Acknowledgments. We thank the anonymous referee and Predehl for comments and kind suggestions which helped to clarify several points. We thank the support from the China postdoctoral science foundation, the Directional Research Project of the CAS under project no. KJCX2-YW-T03, the National Natural Science Foundation of China under grants 10821061, 10733010, and 10725313, and 973 Program of China under grant 2009CB824800.

\section{References}

$\mathrm{Hu}$, J. et al., A new method to determine the distances of variable galactic X-ray sources, Astrophys. J., 614, L45-L48, 2004.

Lee, J. C. and B. Ravel, Determining the grain composition of the interstellar medium with high-resolution X-ray spectroscopy, Astrophys. J., 622, 970-976, 2005.

Lee, J. C. et al., Condensed matter astrophysics: a prescription for determining the species-specific composition and quantity of interstellar dust using X-rays, Astrophys. J., 702, 970-979, 2009.

Ling, Z. et al., A study of the X-ray dust scattering halo of Cyg X-1 with a cross-correlation method, Astrophys. J., 690, 224-230, 2009a.

Ling, Z. et al., Determining the distance of Cyg X-3 with its X-ray dust scattering halo, Astrophys. J., 695, 1111-1120, 2009 b.

Overbeck, J. W., Small-angle scattering of celestial X-rays by interstellar Grains, Astrophys. J., 141, 864-866, 1965.

Predehl, P. and J. H. M. M. Schmitt, X-raying the interstellar medium: ROSAT observations of dust scattering halos, Astron. Astrophys., 293, 889-905, 1995.

Predehl, P. et al., Chandra measurement of the geometrical distance to Cyg X-3 using its X-ray scattering halo, Astron. Astrophys., 357, L25-L28, 2000.

Rolf, D. P., Evidence for the detection of X-ray scattering from interstellar dust grains, Nature, 302, 46-48, 1983.

Smith, R. K. et al., The X-ray halo of GX 13+1, Astrophys. J., 581, 562569, 2002.

Tiengo, A. and S. Mereghetti, Dust-scattered X-ray halos around gammaray bursts: GRB 031203 revisited and the new case of GRB 050713A, Astron. Astrophys., 449, 203-209, 2006.

Tiengo, A. et al., The dust-scattering X-ray rings of the anomalous X-ray pulsar 1E 1547.0-54082009, Astrophys. J., 710, 227-235, 2010.

Trümper, J. and V. Schönfelder, Distance determination of variable X-ray sources, Astron. Astrophys., 25, 445-450, 1973.

Vaughan, S. et al., The discovery of an evolving dust-scattered x-ray halo around GRB 031203, Astrophys. J., 603, L5-L8, 2004.

Weingartner, J. C. and B. T. Draine, Dust grain-size distributions and extinction in the milky way, large magellanic cloud, and small magellanic cloud, Astrophys. J., 548, 296-309, 2001.

Xiang, J., S. N. Zhang, and Y. Yao, Probing the spatial distribution of the interstellar dust medium by high angular resolution X-ray halos of point sources, Astrophys. J., 628, 769-779, 2005.

Yao, Y. S. et al., A new method to resolve X-ray halos around point sources with Chandra data and its application to Cygnus X-1, Astrophys. J., 594, L43-L46, 2003.

Z. Ling (e-mail: lingzhixing@tsinghua.org.cn) and S. N. Zhang 